\title{
Real-Time Neutron Radiography at the IEA-R1m Nuclear Research Reactor
}

\author{
M. O. de Menezes, R. Pugliesi, M. A. S. Pereira, and M.L.G. Andrade \\ Centro do Reator de Pesquisas - CRPQ \\ Instituto de Pesquisas Energéticas e Nucleares - IPEN/CNEN-SP \\ Cidade Universitária, São Paulo-SP, Brasil, CEP 05508-970
}

Received on 30 October, 2002

\begin{abstract}
A LIXI(Light Intensifier X-ray Image) device has been employed in a real-time neutron radiography system. The LIXI is coupled to a video camera and the real-time images can be observed in a TV monitor, and processed in a computer. In order to get the real-time system operational, the neutron radiography facility installed at the IEA-R1m nuclear research reactor of the IPEN-CNEN/SP has been optimized. The most important improvements were the neutron/gamma ratio, the effective energy of the neutron beam, decrease of the scattered radiation at the irradiation position, and the additional shielding of the video camera. Several one-frame as well as computer processed images are presented. The overall Modulation Transfer Function for the real-time system was obtained from the resolution parameter $p=0.44 \pm 0.04 \mathrm{~mm}$; the system sensitivity, evaluated for a Perspex step wedge, was determined and the average value is $0.70 \pm 0.09 \mathrm{~mm}$.
\end{abstract}

\section{Introduction}

Until the beginning of 1997, the neutron radiography (NR) activities at IPEN-CNEN/SP employed imaging systems composed only by conventional X-ray films and solid state nuclear track detectors with metallic(gadolinium and dysprosium), scintillator (lithium fluoride) as well as boron converter screens $[1,2]$. Recently, we have also used a LIXI (Light Intensifier X-ray Image) device to obtain real-time images. In such device, neutron scintillations are amplified by a light intensifier and a video camera captures the resulting light, given rise to an analog image which can be seen in the TV monitor, and digitized. The resulting one-frame images can be processed in the computer.

The present real-time system has been characterized in terms of its overall Modulation Transfer Function(MTF). In addition it was also verified the system sensitivity to discern thickness.

\section{Experimental}

The experimental NR facility employed in this work is installed at the beam-hole \#08 of the 2MW IEA-R1m nuclear research reactor. In order to get the real-time system operational, the original facility designed in 1987, has been improved. Presently, the external shielding has a large internal free space $(2 \times 2 \times 2 \mathrm{~m})$ providing several irradiation positions and a negligible scattered radiation contribution at the irradiation position. The new Cd-borated paraffin divergent collimator, $1.5 \mathrm{~m}$ length leads to a maximum L/D ratio of about 110. The bismuth filter, $25 \mathrm{~cm}$ thick assures a low gamma radiation contribution to the image formation and reduces the effective energy of the neutron beam. The characteristics of the neutron beam depend on the irradiation position inside the shielding, and are shown in Table 1 for the positions where the neutron flux is maximum and minimum.

\begin{tabular}{|c|c|c|}
\hline neutron flux & $110^{6} \mathrm{n} / \mathrm{s} . \mathrm{cm}^{2}$ & $1.610^{5} \mathrm{n} / \mathrm{s} . \mathrm{cm}^{2}$ \\
\hline $\mathrm{n} / \gamma$ & $410^{6} \mathrm{n} / \mathrm{cm}^{2} . \mathrm{mrem}$ & $210^{6} \mathrm{n} / \mathrm{cm}^{2} . \mathrm{mrem}$ \\
\hline $\mathrm{L} / \mathrm{D}$ & 55 & 110 \\
\hline effective energy & $7 \mathrm{meV}$ & $7 \mathrm{meV}$ \\
\hline
\end{tabular}

Table 1: Main characteristics of the neutron beam

The LIXI is a device with a $5 \mathrm{~cm}$ diameter input neutron scintillator, which is coupled to a light intensifier(gain $10^{4}$ ). The amplified image is captured by a high sensitivity - 0.01 lux CCD (Charge Coupled Device) video camera which is attached to a video cassette recorder (VCR), to a television (TV) monitor as well as to a computer. During irradiation, the LIXI scintillator is positioned perpendicularly to the neutron beam and the video camera perpendicularly to the former. Both are installed inside a light tight box having a $45^{\circ}$ plane mirror. The camera is protected against fast neu- 
trons by means of an additional borated-paraffin shielding. The TV monitor, the VCR and the computer are installed outside the facility shielding. A schematic diagram of the real-time system is shown in the Fig. 1.

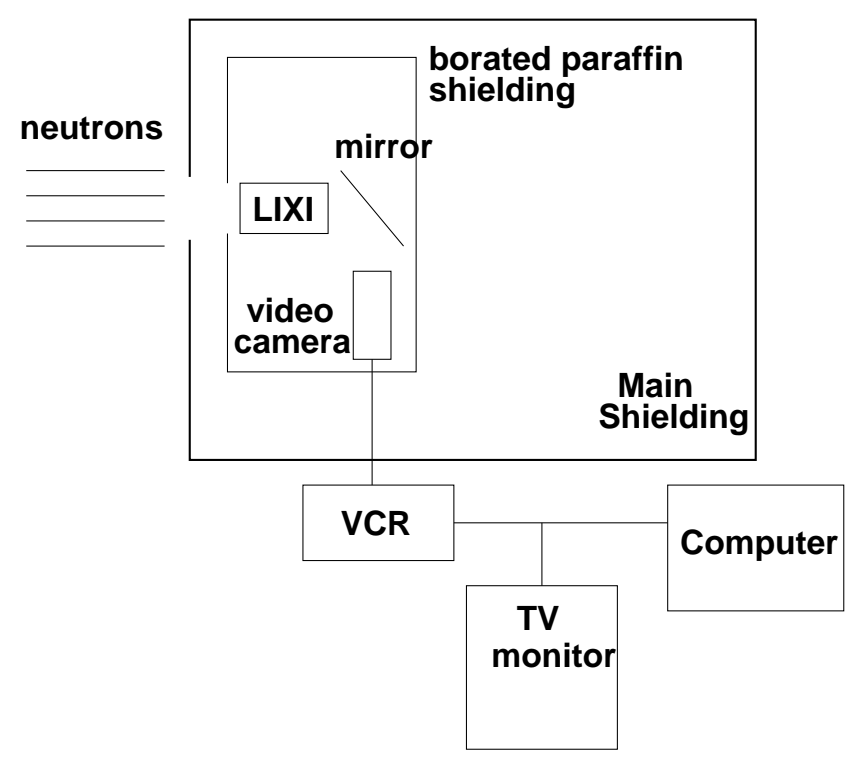

Figure 1. Schematic diagram of the real-time system.

The real-time images were obtained by positioning the LIXI device where the neutron flux is maximum. The images coming from the video camera can be visualized in the TV monitor and stored in a cassette tape or in the computer hard disk. The images are digitized by means of a Targa-1000 frame grabber at a rate of 30 frames/sec with 256 gray levels, installed in the computer. Because of hardware limitations, the image processing is not performed in real-time. Several experiments have been carried out in order to observe static and dynamic objects such as cadmium and gadolinium strips, Perspex step wedge, water movement inside an aluminum cavity. The obtained one-frame images as well as the processed ones are shown in Fig. 2 and Fig. 3 , respectively.

The MTF was determined by a conventional method [3], by scanning the image of a neutron opaque material(gadolinium strip), which has been irradiated in a tight contact with the LIXI scintillator. An arctan Edge Spread Function (ESF) has been fitted to the resulting pixel intensity distribution. By differentiating the ESF, the Line Spread Function (LSF) is obtained and its Fourier transform gives the resulting MTF:

$$
M T F=C \cdot \exp (-2 \pi f / p)
$$

where $\mathrm{C}$ is a constant, $\mathrm{f}$ is the spatial frequency (lines $/ \mathrm{mm}$ ) and $\mathrm{p}$ is the resolution parameter. In Fig. 4 the system MTF corresponding to an average of 75 frames is shown.

The system sensitivity to discern thickness is related to several factors, such as: material thickness $(x)$; total macro- scopic cross section $\left(\Sigma_{T}\right)$; imaging system performance; exposure $(E)$ defined as the product of neutron flux by irradiation time $(\Phi t)$.

Since the neutron transmisstion is governed by the well known exponential law

$$
\Phi=\Phi_{0} e^{-\Sigma_{T}(v) x}
$$

and that the light intensity generated in the scintilator (bright $B$ ) is a linear function of the exposure $(B \propto E)$, we can write:

$$
B=B_{0} e^{-\Sigma_{T} x}
$$

Considering that the gray levels $(g)$ of the digitized image and the gray levels of the analog image are related by a $\gamma$ factor given by [6]:

$$
\gamma=\frac{\frac{\Delta g}{g}}{\frac{\Delta B}{B}}
$$

the sensitivity obtained for the digital image can be given by:

$$
\Delta x=\frac{\Delta g}{\gamma g \Sigma_{T}}
$$

where $\Delta g$ is the minimum discernible change in the gray levels.

The system sensitivity to discern thickness has been evaluated by imaging a Perspex step wedge, with thicknesses ranging from 2 to $15 \mathrm{~mm}$. The step wedge was irradiated as close as possible to the LIXI device. From a 75 frames averaged image we performed gray level readings (300 pixels) in each step; these readings were averaged arithmetically and the resulting values as a function of the thickness are shown in Fig. 5.

\section{Discussion}

For similar real-time systems, high level noisy and low contrast analog images are frequently obtained $[4,5]$. However, the present analog images are good in contrast and resolution. This can be a consequence of the low effective energy of the present neutron beam which leads to a high effective cross section for the materials and to a high neutron to photon conversion efficiency of the LIXI scintillator.

In the Fig. 2(a, b, c) and Fig. 3(a, b, c) the one-frame images as well as the processed ones for the static objects are shown. In this case, frame averaging, edge and contrast enhancement has been applied and improvements in image quality have been obtained.

As already mentioned, because of hardware limitations, only one frame of the dynamic object, has been processed. In this case, neighbour pixel averaging as well as contrast and edge enhancement, have been applied. By comparing the Fig. 2d with Fig. 3d, the processing has provided improvements of image quality to observe details of the water turbulence inside the aluminum cavity. 
a.

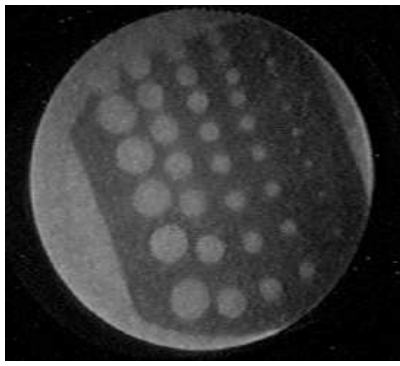

c.

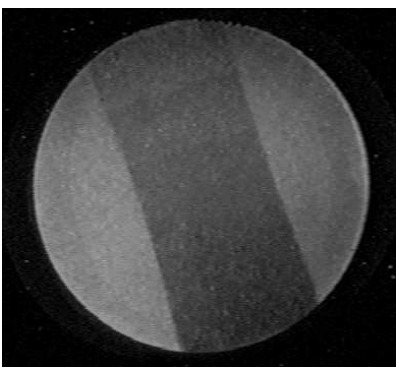

b.

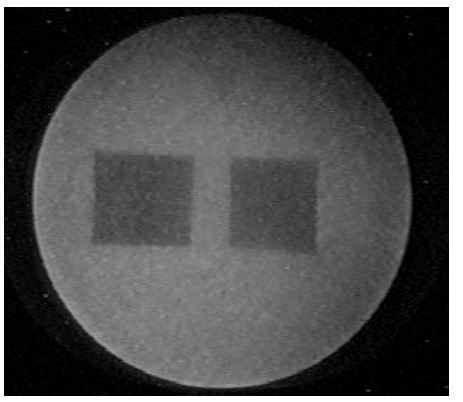

d.

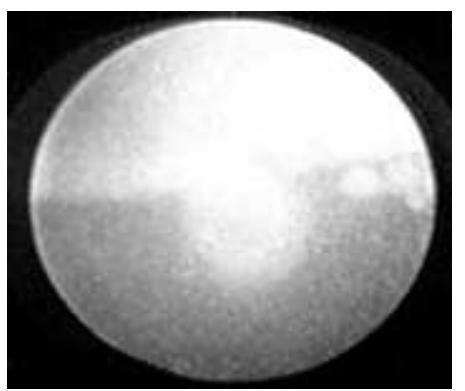

Figure 2. One-frame images: a. cadmium strip; b. gadolinium strip; c. Perspex step wedge; d. water movement.

a.

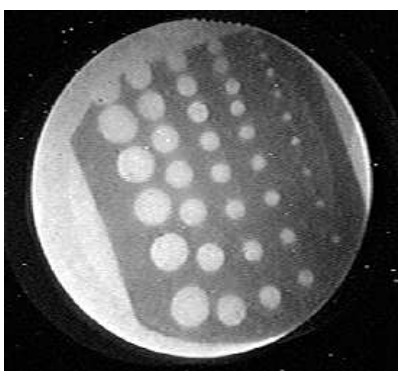

c.

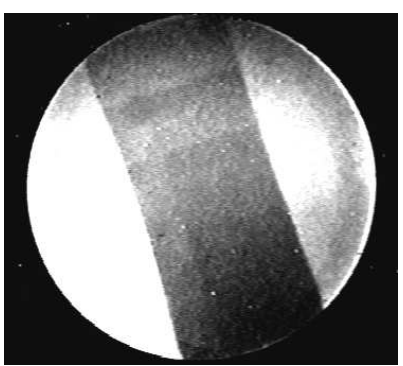

b.

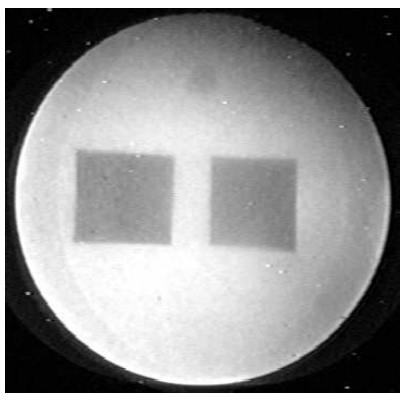

d.

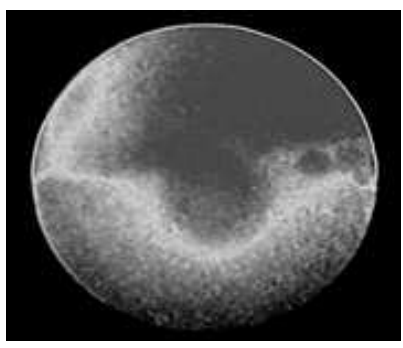

Figure 3. Processed frames: Averaging, edge and contrast enhance: a. cadmium strip; b. gadolinium strip; c. Perspex step wedge; d. water movement. 
In the Fig. 4 the overall MTF as a function of the spatial frequency (lines $/ \mathrm{mm}$ ) is shown. The image used for the MTF measurements was 75 frame averaged. The resolution parameter obtained for this image is $p=0.44 \pm 0.04 \mathrm{~mm}$.

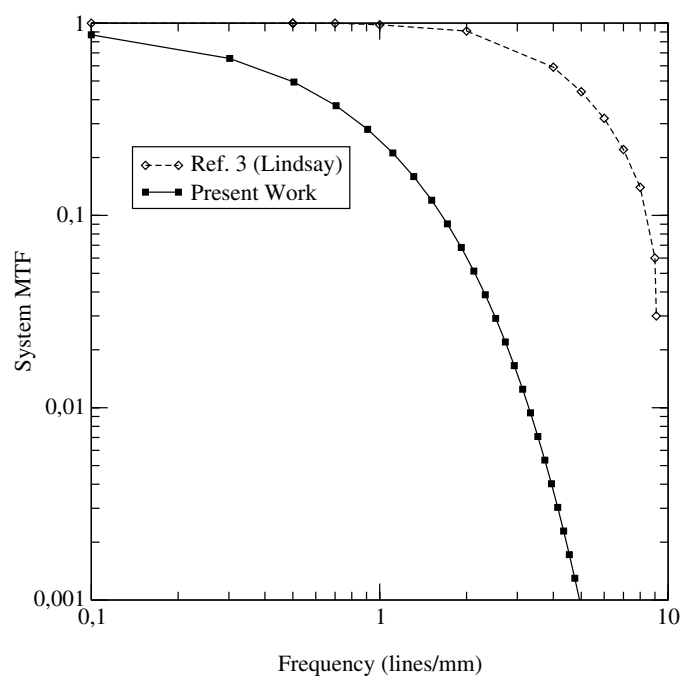

Figure 4. Modulation Transfer Function for the present system and from literature [3].

Although a noise reduction has been obtained, the present MTF when compared with that from literature [3], shows a faster decay after frequency of 0.3 lines $/ \mathrm{mm}$, denoting a worse image quality for the present system.

Concerning the system sensitivity, the greatest variation in gray level, that is, the greatest standard deviation for the readings of gray levels of the steps is $\Delta g=9$. Using $\gamma=1$ [7] and the total macroscopic cross section of Perspex $\Sigma_{T}=0.94 \pm 0.11 \mathrm{~cm}^{-1}$, the minimum discernible thickness change will be given by:

$$
\Delta x=\frac{9}{0.94 g}
$$

For $g_{\max }, g_{\text {med }}$ and $g_{\min }$ shown in Fig. 5, the corresponding $\Delta x$ are:

- $g_{\max }=218 \pm 8$, so $\Delta x=0.44 \pm 0.05 \mathrm{~mm}$

- $g_{\text {med }}=137 \pm 8$, so $\Delta x=0.70 \pm 0.09 \mathrm{~mm}$

- $g_{\min }=57 \pm 3$, so $\Delta x=1.68 \pm 0.22 \mathrm{~mm}$

The average sensitivity value obtained for the Perspex, $0.7 \mathrm{~mm}$, is superior to those obtained from real-time systems using thermal neutron beams [3], reflecting the high neutron-light converter screen conversion efficiency for the neutron effective energy of $7 \mathrm{meV}$ (see Table 1). The sensitivity value is also comparable to those obtained for film neutron radiography $(0.45 \mathrm{~mm}[8])$, which often have higher sensitivity.

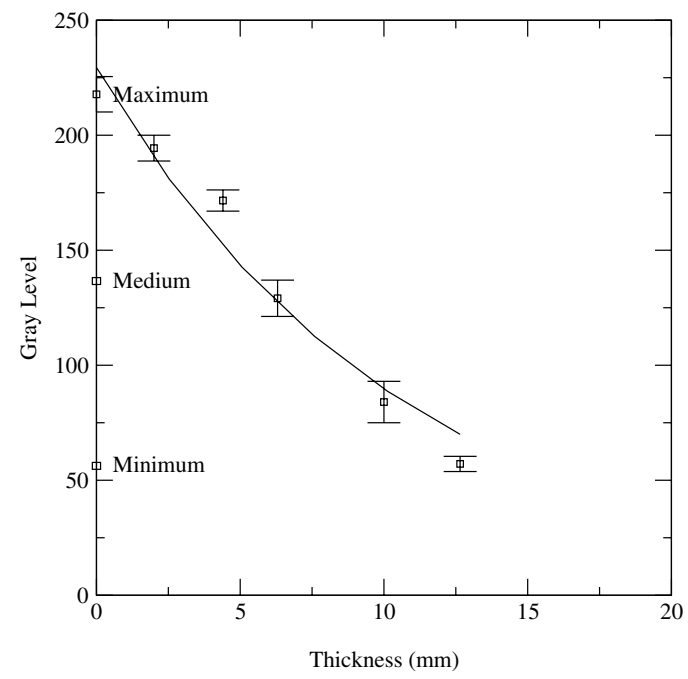

Figure 5. Perspex step wedge gray levels distribution as a function of thickness.

Acknowledgments: The authors would like to acknowledge Fundação de Amparo à Pesquisa do Estado de São Paulo for the financial support of this project.

\section{References}

[1] R. Pugliesi, M.O. de Menezes, M.L.G. Andrade, M.A.S. Pereira, M.S.J. Maizatto, Nucl. Instr. and Meth. A 424, 248 (1999).

[2] R. Pugliesi, M.A.S. Pereira, M.A.P.V. de Moraes, M.O. de Menezes, Appl. Rad. Isotopes 50, 375 (1999).

[3] J. T. Lindsay, Ph.D. Thesis, University of Missoury, May 1983.

[4] S. Fujine, K. Yoneda, K. Kanda, Nucl. Instr. and Meth. A 228, 541 (1985).

[5] J. T. Lindsay, J. D. Jones, C. W. Kauffman, B. van Pelt, Nucl. Instr. and Meth. A 242, 525 (1986).

[6] L. E. Bryant and P. McIntire, editors Nondestructive Testing Handbook, Radiography and Radiation, Vol. 3, American Society for Nondestructive Testing, Columbus, Ohio, 1985.

[7] Precise Optics, Inc., 239 South Fehr Way, Bay Shore, NY., Instruction Handbook PVC525 PVC625 - High Performance CCTV Camera.

[8] M. O. de Menezes, Master Thesis, Universidade de São Paulo, USP, 1994. 